\title{
Study on "A Thousand Li of Rivers and Mountains" by Wang Ximeng
}

\author{
Yurong $\mathrm{Ma}^{1, *}$
}

${ }^{1}$ Faculty of Innovation and Design, City University of Macau, Macau 999078, China

${ }^{*}$ Corresponding author. Email: 739123477@qq.com

\begin{abstract}
This article takes the Northern Song Dynasty painter Wang Ximeng's "A Thousand Li of Rivers and Mountains" as the object of inquiry. Based on the overview of the paintings, it analyzes the color features of the green landscapes and the beauty of the artistic conception displayed in the pictures, and attempts to divide the picture from the perspective of music. This huge scroll reproduces the magnificent scenery of the mountains and rivers during the Northern Song Dynasty. "A Thousand Li of Rivers and Mountains" is the pinnacle of Chinese green landscape paintings, and it has extremely high historical and aesthetic value in the field of Chinese painting.
\end{abstract}

Keywords: Wang Ximeng, A Thousand Li of Rivers and Mountains, green landscape, color, artistic

conception

\section{INTRODUCTION}

The work "A Thousand $\mathrm{Li}$ of Rivers and Mountains" is created by Wang Ximeng from Song Dynasty, who is a mysterious recluse in the history of art. Falling in relentless time, he is remembered by the world because of a marvelous painting. Eighteen-yearold Wang Ximeng was an apprentice in an imperial painting institute, but later he was assigned to the document library. The perspicacious Emperor Huizong of Song Dynasty who was once served by Wang Ximeng thought that he was worth teaching. So the emperor taught Wang Ximeng his own painting skills. With the improvement of Wang Ximeng's painting ability, a masterwork was painted by him within half a year. Unexpectedly, the painting is the only work handed down of the talented youth, without record in the painting literature later. And the rest of his works do not exist in the world.

\section{A BRIEF DESCRIPTION OF THE GREEN LANDSCAPE BY WANG XIMENG}

"A Thousand Li of Rivers and Mountains", in the collection of the Palace Museum in Beijing, is voluminous ink and pigment on silk. The painting is in the length of $51.5 \mathrm{~cm}$ vertically, and $1191.5 \mathrm{~cm}$ horizontally. With a poem written by Qianlong at the beginning of the painting and postscripts by Caijing from Song Dynasty at the end, the work tells the creating time, age and identity of the painter. Monk Fuguang, a famous calligrapher and painter in Yuan Dynasty, wrote a postscript, admiring "A Thousand Li of Rivers and Mountains". "Since I was eager to learn from others, I have witnessed this painting for nearly a hundred times. I cannot still look through all the details in this work because I can always find some now information. And it has bright colors and magnificent layout, which may make Wang Jinqing and Zhao Qianli ashamed when seeing this marvelous painting. Among all the paintings on green landscapes, this work can be unique and the leader for thousands of years ". Thanks to the aesthetical ideal and pursuit of magnificence by the royal, landscape paintings of green and gold colors were enjoyed popularity among the royal children. "A Thousand Li of Rivers and Mountains" is famous for bright blue green. After over 900 years, except that the silk is slightly darker, the color is still as bright as the new, which is hard to be exceeded.

Green landscape is one of the patterns of manifestation of meticulous heavy color painting. As one of the art varieties of Chinese landscape painting, the pattern of green landscapes drawn with the early outline was showed in the works of the painter $\mathrm{Gu}$ Kaizhi. And green landscape is also a kind of painting with relatively early maturation time. Green landscapes portray the splendid world with mineral green and azurite. Compared with modern chemosynthetic pigments, while coloring, natural mineral substances whose colors are not easy to change are characterized with wide coverage, purity and brightness. The paintings drawn with mineral substances are magnificent and splendid. The criterion of distinguishing different kinds of blue green is the proportion of water and ink. The dark green is rich in sketches, with few chapping methods, heavy colors and 
strong feature of decoration. The light green is just a thin layer of green on the basis of the light color of ink, cyan and green intersecting with each other, which enjoys a decisive position in the field of ancient painting. "A Thousand Li of Rivers and Mountains" dominated by the bright cyan and green is praised as "being unique for thousands of years" and "being the brightest among all the other works" and it also contributes a lot in the history of Chinese landscape painting.

\section{THE COLORING FEATURES OF WANG XIMENG'S LANDSCAPE PAINTING}

The coloring of Chinese green landscapes existing in the world now is ink painting dominated. Represented by Huang Gongwang and Wang Meng, the elegant "landscape in light purple" is relatively common, fewer are in blue green. Different from ink landscape, "A Thousand Li of Rivers and Mountains" is one of the coloring landscapes. The painting has been collected well because of the natural mineral pigments which are the secret of colorfastness for thousands of years. Xie He from the Southern Qi Dynasty said in "Artistic Quality" that "six laws" should be regarded as the evaluation criteria of paintings, whose details are " vividness, forcefulness, graphicness, colorfulness, structuredness, iconicity " in which "colorfulness" means the basic principle of the coloring of Chinese paintings.[1]

When painting "A Thousand Li of Rivers and Mountains", Wang Ximeng broke through the traditional style of ink painting, and boldly blended the north and south style. At the time of rendering, the sense of cloudiness and mist in Jiangnan region created by the southern Dong Yuan, famous for "plain innocence, brand new but tangy" [2], was combined with the magnificent scenery of the northern mountains and waters represented by $\mathrm{Li}$ Cheng. The contents of the subject in the picture are greatly enriched by human activities such as hill living, traveling, fishing, and gathering. The hills run along the south, feeling like a fairyland. The image of the mountains in the north has a strong sense of grandeur. Mountains, rocks and hills are outlined, where heavy ink was applied, and then some parts are scrubbed with lines. The texture, volume and structure of the mountains and rocks are also revealed through color rendering or dyeing step by step. Sometimes argillious gold ore was used to draw the outlines, stone grain and pavilions to increase the feeling of magnificence. It is called "Golden Landscape" because of the color gold which is not applied in the "green landscape". Enjoying the "A Thousand Li of Rivers and Mountains" more clearly, we can find distinct coloring and layering of lines. From one point of view, compared with the monotonous superimposition and simple flat painting techniques appearing in the Sui and Tang dynasties, there have been significant advances. The magnificence of the mountains is presented through being layered with high-purity mineral blue and green. The high purity and heavy colors make the entire long-length painting particularly bright and brilliant.

The painting "Ode to the Goddess of the Luo River" drawn by the painter Gu Kaizhi from the East Jin Dynasty has the feature that "humans are more important than mountains and water is not too much" [3]. The proportions of landscape figures in the Song Dynasty are different from those in the early paintings, where humans and the sky and earth weigh the same. In the painting, there are many bean-sized people with different poses. Ant-sized figures do their won work, which is presented vividly. Some are coated with thick white powder, which is also obvious in the thick, green mountains and rocks, and some are elaborately sketched. While paining, different coloring techniques are used, with blue and green as the main tone of the picture, and ochre as the background. As a result, the whole frame is not monotonous, but with a sense of layering, which can be said to be as radiant as gemstones. "Flying birds can be in the tendency to soar with only a light sketch. The light water waves are drawn line by line, on which the fishing boat swings, adding motion to the picture. Overviewing the whole frame, it does not lose the magnificent state and magnificent momentum. " [4]

With the painting skill of layering with colors instead of ink, "A Thousands $\mathrm{Li}$ of Rivers and Mountains" describes magnificent and expansive scenery. This kind of long-length painting is rarely seen among the masterpieces.

\section{THE MUSICIANSHIP IN THE GREEN LANDSCAPE PAINTINGS OF WANG XIMENG}

Fine arts and music are both forms of art. Music is the consolidated "painting", while painting the fairytale "music". "A Thousand Li of Rivers and Mountains" ("Fig. 1") is like a complete symphony, which can be divided into six parts: section, prelude, rise, development, climax, fall and end. The mountain in the painting is running for thousands of miles, with high and low levels, and contains a strong sense of rhythm and order. When creating this work, Wang Ximeng obviously applied midpoints, lines, and faces in calligraphy to compose works with a sense of musical rhythm and bring new life to the paintings.

The painting begins with the falling shallow mountain as the prelude. At the beginning of the painting, the mountain with pointed and dome peak runs upwards to two thirds of height of the whole frame. Later, the mountains fall back, and the hills are scattered around, which are connected by wavy wooden 
bridges. On the left of these bridges, the peaks of all the mountains leans slightly to the right, like making a bow with hands folded in front. In the rising part, the picture scroll is mainly composed of shallow mountains. With mountains gathering, some are towering or standing, some tortuous, and some magnificent. The mountain in the middle gradually becomes higher, meaning that it is the center of the section. In the prelude, the mountain here gradually rises, and the highest peak occupies four fifths of the picture, forming a clear center. The endless mountains on the left and right interact with each other, and the surging marvelous scene is a hint to the appreciator that the climax of the picture is about to start. The climax of the painting means that it is the most exciting part in the whole work. With the main mountain almost rising to the top of the painting as a sign, there are some great differences between the main mountain and the surrounding hills. The smooth lines, heavy colors, and delicate texture are very visually outstanding. In the falling part, the picture gradually returns to be gentle, and the overall mountain gradually dies away, showing a downward trend. The mountain on the left is slightly higher, and the mountain falls back quickly. The ensuing is the end of the painting, where the peaks of the picture are gradually picking up. Especially, the rise of pointed and domed mountains echo with those at the beginning of the painting, forming a closed structure. The painting ends with a flat and distant view, and the surface of the river also extends into the distance. Grazing in the mountains, fishermen spreading nets on the shore, the whole frame consists of the rural life of ordinary people.

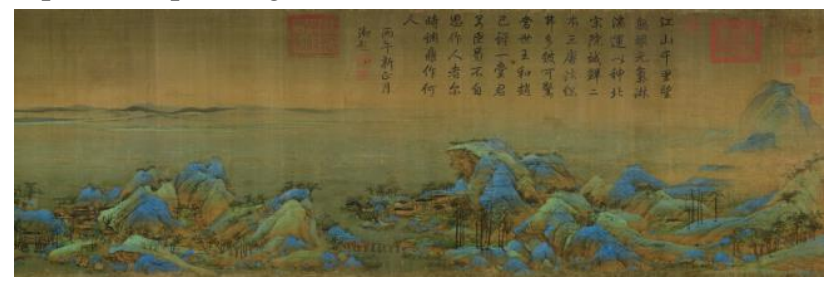

Fig. 1. A Thousand Li of Rivers and Mountains (part) (From the Palace Museum).

Musical notes and musical notation are integrated into music, lines and colors into calligraphy and paintings. If lines are regarded as stave, color can be seen as notation, which can be said that "there are paintings in music and sound in paintings". Wang Ximeng's "A Thousand Li of Rivers and Mountains" is collected as one of China's top ten masterpieces. And Autumn in Rivers and Mountains painted by Zhao Boju, another landscape painter in the same era, can match with the work of Wang Ximeng. Same as A Thousand Li of Rivers and Mountains, in the long scroll of silk, Zhao Boju's painting is characterized by details and changes in microscopic view. From a macro perspective, the layout is more grand. Although the colors are thick and rich, it is still fresh and elegant, with the style of the Song Dynasty Academy of Painting.

\section{THE CHARACTERISTICS OF ARTISTIC CONCEPTION OF WANG XIMENG'S GREEN AND LANDSCAPE}

The beauty of artistic conception is based on real life, whose roots are nature and reality. Of course, it is also an indispensable and important part of Chinese traditional painting, which contains the artist 's unique aesthetic interests and ideas. It is important that the artistic conception in a painting exists, the artistic conception is distinct, or it allows the appreciator to be immersed in the scene to experience the unique blending of scenes and the poetic space in traditional
Chinese painting, which is also an extremely high artistic state pursued by painters of all ages.

Wang Ximeng's "A Thousand Li of Rivers and Mountains" is far-reaching in the way of expressing artistic conception. In his masterpiece, the image of space of feasibility, expectation, traveling, and habitability is crucial. It can express the image of peaceful world: the distribution of mountains and forests reflects the order between an emperor and courtiers, gentlemen and snobs; the mountain landscape is endless and steep, but it does not show the pass of the city defense, but emphasizes the leisurely elegance of the scholars and the livelihood of the people; the clear water and the blue waves are not only the performance of the season, but also the symbol of peace. The waves in A Thousand Li of Rivers and Mountains are painted with high-purity mineral green, stressing the state of clean water. Emperor Huizong of Song Dynasty claimed to be the emperor of Taoism and paid great attention to auspiciousness. The two words "clean water" appears repeatedly in "Song History · Huizong Benji", which is closely related to "World Peace". In the late Northern Song Dynasty, with Song Huizong's New Deal reform, "A Thousand $\mathrm{Li}$ of Rivers Mountains" also reflected the political and religious influence of art, showing a vibrant river and mountain, and a thriving society.

The "artistic conception" in Chinese painting is one of the traditional aesthetic ideas in the field of painting, which is also one of the senior features of literary images in the field of literature. This supreme aesthetic 
criterion has become a unique national art form in Chinese painting after years of baptism of time. In the work A Thousand Li of Rivers and Mountains, Wang Ximeng's superb painting skill shows the beauty of the artistic conception of Chinese painting to the world. The uniqueness of Chinese painting is vividly displayed, demonstrating the vitality of Chinese art.

\section{CONCLUSION}

"Literature and art are advance bugle of the era, a representative of the era, and a leading spirit of the era."[5] "A Thousands Li of rivers and mountains" is hailed as a treasure in the history of China's green landscape paintings. It gathers the essence of the paintings in Tang and Song dynasties and the color of China's five elements. The painting is thousands of miles away but with sentiment. The magnificence of mountains and rivers in China is vividly displayed in Wang Ximeng's work. He expresses it with general exquisite techniques, brilliant colors and meticulous style of using brushes, precipitating the Chinese culture for thousands of years. When the paintings were completed, it was a piece of artistic legend of mountains and rivers. Existing for thousands of years, the painting is greener when appreciated today. With thousands of miles of rivers and mountains, culture will last forever in the long river of time.

The rapid development of science and technology not only provides new means of expression for art, but also expands the space of art expression. "A Thousands $\mathrm{Li}$ of Rivers and Mountains 3.0" digital art exhibition was exhibited at the Macao Museum of Art, presented to the world in another way. The development of science and technology has given the opportunity for the combination of culture and art, art and joy, proclaiming a new way of life in traditional aesthetics. Feel the instant of time and the eternity of universe in the painting; experience the pleasure of enjoying the landscape and the inner peace when imaging traveling in the mountains. Comprehend the beauty of harmony and the image of freedom in Macau, a maritime lotus.

"A Thousand $\mathrm{Li}$ of Rivers and Mountains" is colorful and charming, is the magnificence of the shift of stars, is the cycle of sun and moon, and is also the tone of the ensemble of mountains and rivers. " A Thousand Li of Rivers and Mountains" is unique among all the paintings, shining brightly in the stars with the light of moon. "The highest state of art is to be inviting, let people's souls be baptized, and let people discover beauty."[6] Today, it is still influencing the later art practitioners. With the shining light of brilliant green, this long green landscape scroll is of unique historical value.

\section{References}

[1] Ge Caijing, and Luo Zhenyu, "Six Lows by Xie He and Bonsai". Lvhua Yu Shenghuo. 2001, (06): 12.

[2] Yang Yu, "On Famous Artists - Thoughts on the Theory of DongYuan". Art Education. 2012, (06): 111.

[3] Meng Xianping, "Criticism on the misuse of Water Cannot be Too Much and People Should Be More Important Than Mountains - A Viewpoint on Reflecting Study of Ancient Art History". Journal of Nanjing Arts Institute: Fine Arts and Design. 2010, (4): 102-107.

[4] Liu Xuewei, "Painting Institute in Song Dynasty and A Thousand Li of Rivers and Mountains by Wang Ximeng". Literary Contentions. 2010, (24): 61-62.

[5] "Finding the Position and Position of Literary and Artistic Work in the New Era" [N]. Guangming.com, July 31, 2018.

[6] Chen Zehuan. Times and nationality: the academic construction of cultural self-confidence [J]. Journal of Shenzhen University: Humanities and Social Sciences Edition, 2018, v.35; No.172 (04): 5-11. 